\title{
80 PLUS ONE \\ Zwei oder drei Publikationen aus dem Godard-Jahr 2010 sowie zahlreiche Internetseiten und einige Filme
}

von FLORIAN KRAUTKRÄMER

Antoine de Baecque, Godard, Paris (Bernard Grasset) 2010.

Bernard-Henry Lévy, Godard est-il antisémite?, in: La Règle du feu, Heft 45, 2 I. Jg., Januar 201 I, I99-252.

Bernd Kiefer, Thomas Koebner, Fabienne Liptay (Hg.), fean-Luc Godard, München (edition text + kritik) 2010 (Film-Konzepte, Bd. 20).

So viel Godard war selten - zumindest nicht, seit seine Filme nicht mehrim Kino gezeigt und außerhalb von Cannes und Hochschulen auch nicht mehr gesehen werden, seit gut zwanzig Jahren also. («If only he had stopped while he was ahead. That would have been sometime in the 1970 s. Maybe the 1980s. For sure, the 1990s. Without a doubt, before he made his Cannes entry, Film: Socialisme.») ${ }^{\mathbf{1}} 2010$ war das Godard-Jahr. Bereits seit Cannes 2009 kursierten die im Schnelldurchlauf abgespielten Trailer seines neuen Films. Ein Jahr später wurde Film socialisme in der Nebensektion Un certain regard präsentiert. Die Hauptaufregung: Kommt Godard oder nicht? Wie schon häufiger hielt sich Godard durch Abwesenheit im Gespräch - er kam nicht. ${ }^{2}$ Dafür gab es das pressbook zum Download, ${ }^{3}$ inklusive Interview: «Q: static shots only? A: the chemist doesn't do tracking shots in front of his microscope nor petrol companies when drilling into the sea bed.» Und die Aufregung blieb: Godard sollte am 13. November der Ehrenoscar verliehen werden - auch hier war man sich lange unsicher, ob er diesen persönlich in Empfang nehmen würde: Tat er dann aber nicht («Would you go all that way just for a bit of metal?») ${ }^{4}$

\section{Godard est-il antisémite?}

Doch das Rätseln über Godards Anwesenheit wurde bald durch eine viel größere Debatte abgelöst: «Godard est-il antisémite?». Für diesen möglichen Antisemitismus, der, wenn überhaupt, sich hinter dem kaum cachierten AntiZionismus verbirgt, ${ }^{\mathbf{5}}$ finden sich in mehreren GodardFilmen Hinweise; Stein des Anstoßes bei der aktuellen Debatte war jedoch eine Szene aus Ici et Ailleurs (Jean-Luc Godard, Anne-Marie Miéville, F 1976), gegen den schon im Jahr seiner Veröffentlichung verschiedene zionistische Gruppen in Paris protestierten (vgl. de Baecque, S. 530). Besonders problematisch ist im Film die Szene, in der er das Bild Adolf Hitlers auf das Golda Meirs treffen lässt. Obwohl der Film kaum gesehen wird, kam dieser Ausschnitt erneut in die Diskussion, unter anderem weil er in Alain Fleischers Dokumentarfilm Morceaux de conversation avec Jean-Luc Godard (F 2007), der 2009 in die Kinos kam und Anfang 2010 auf DVD erschien, prominent diskutiert wurde. In einer sehr langen Episode unterhält sich Godard dort mit dem ehemaligen Redakteur der Cahiers du Cinéma, Jean Narboni, zunächst über den Film Notre Musique (JeanLuc Godard, F/CH 2004), bevor sie eben auf diesen Ausschnitt von Ici et Ailleurs zu sprechen kommen ( $\mathrm{N}$ : Ça reste rugueux. G: Ah non, je n'y trouve rien à changen»). Narboni hinterfragt die Analogie zwischen Hitler und Golda Meir, woraufhin sich Godard auf die Position zurückzieht, es gäbe keine Objektivität der Bilder - der eine sähe das eben so, der andere so. Auch Daniel Cohn-Bendit setzte sich anlässlich eines Artikels zu Godards achtzigstem Geburtstag mit der Frage auseinander, ob dieser ein Antisemit sei, kommt aber ebenfalls zu keiner Antwort: «[D]iese Frage ist nicht lösbar.» ${ }^{6}$ 
Anfang 2011 stellte Bernard-Henri Lévy auf dem Titel der fünfundvierzigsten Ausgabe seiner Zeitschrift La Règle du Jeu erneut die Frage nach Godards Antisemitismus, aber die Frage blieb rhetorisch («désolé si l'on ne se sent pas, du coup, plus avancé», S. 252). Dafür berichtet er anhand von Briefwechseln zwischen ihm und Godard über zwei Fernsehsendungen, die 1999 und 2006 unter Beteiligung der beiden hätten entstehen sollen und in Planung und Produktionsvorbereitung sogar recht weit gediehen waren. ${ }^{7}$ Am ehesten trägt noch der vorletzte Brief Godards an Lévy zum Thema bei, der dann auch das Filmprojekt Terre Promise zum Platzen brachte: Der Plan sah vor, dass BHL \& JLG gemeinsam nach Israel reisen sollten, kurz vor Abfahrt schlug Godard jedoch vor, dass man sich dabei auch mit Tariq Ramadan unterhalten müsse - eine Unmöglichkeit für Lévy, der sich mit Ramadan bereits zuvor öffentlich auseinandergesetzt und ihn als Faschisten bezeichnet hatte (vgl. La Règle du Jeu, S. 251). Doch die veröffentlichten Dokumente sind auch jenseits der spekulativen Fragestellung von Interesse, denn in den bekannten Biografien finden sich keine ${ }^{8}$ oder zumindest kaum Hinweise $^{9}$ auf diese Episoden.

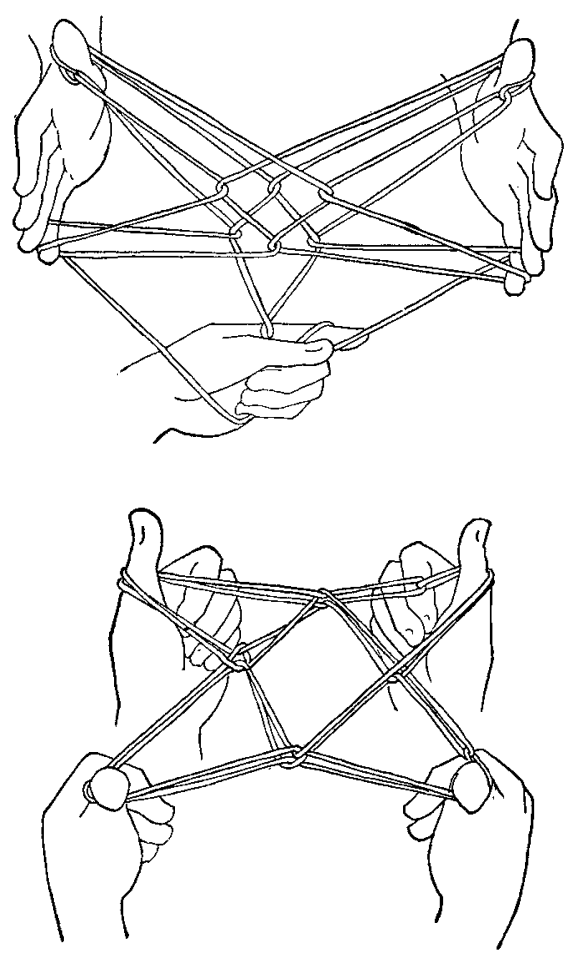

Die beiden Fernsehprojekte sind die Fortsetzung einer Debatte zwischen Claude Lanzmann ${ }^{\mathbf{1 0}}$ und Godard über Bilder aus den Konzentrationslagern, die Mitte der goer Jahre begann und auch bei de Baecque nachgezeichnet wird. Lanzmann hatte in einem Interview 1994 davon gesprochen, dass er, sollte er einen Film mit Aufnahmen von Tötungen in der Gaskammer gefunden haben, diesen vernichtet hätte. Godard hatte 1985 bereits gesagt, er sei sicher, dass die Deutschen die KZs gefilmt hätten und die Aufnahmen sich irgendwo befinden müssten. 1998 wiederholte er diese Ansicht, mit der Präzisierung, dass er zusammen mit einem guten Journalisten innerhalb von 20 Jahren diese Archive finden würde. Eine unnötige Vermutung, da sie die Bild-Diskussion um die Shoah in eine Beweislogik zwängt, und die Gérard Wajcman zur Frage veranlasste, was es denn dann bedeuten würde, wenn man die Bilder doch nicht fände (S. $7^{6}{ }_{3}$ ff.). ${ }^{11}$ Das erste Fernsehprojekt war als eine Art Aussprache Godards und Lanzmanns geplant, bei der Lévy als Vermittler fungieren sollte.

\section{Godard par de Baecque}

Der Verdienst von Antoine de Baecques umfangreicher Biografie liegt darin, auch derartigen Ereignissen Raum zu bieten und sich nicht allein darauf zu beschränken, die biografischen Details um die Filme herum anzuordnen. Er führt zudem eine Fülle von Quellen sowohl zu den einzelnen Filmen als auch den unterschiedlichen Stationen Godards an, die, auch wenn sie anders als in Alain Bergalas zweibändiger Materialsammlung Godard par Godard ${ }^{12}$ nur in Auszügen wiedergegeben werden können, das Buch zu einem wichtigen Baustein der Godardforschung werden lassen. Zwar bleibt es eine Biografie, die kaum neue inhaltliche und theoretische Auseinandersetzungen mit den Filmen bietet, auch die biografischen Aspekte sind hinlänglich bekannt. De Baecque zeichnet das Portrait eines teilweise unsicheren und suizidgefährdeten Künstlers, der vor allem in seiner Spätphase an seiner Einsamkeit zu leiden hat. Es sind aber die zahlreichen von de Baecque herangezogenen Interviews, Filmkritiken und Dokumente, die hier den Unterschied machen, weswegen das Buch letztendlich als Künstlerbiografie weniger gut lesbar ist als die von Colin MacCabe oder Richard Brody. ${ }^{13}$ Dafür fördert De Baecque durch seine Recherchen aber eine Menge ergänzender Informationen zutage, was gerade bei den bisher weniger bekannten Filmen, wie denen der Groupe Dziga Vertov, ${ }^{14}$ hilfreich ist. Zwar liegt de Baecques Schwerpunkt auf der Nouvelle Vague vor 1968, 
aber durch die penible Quellenarbeit kann de Baecque einige etablierte Perspektiven teilweise verschieben. So zeigt er anhand der Materialien zu den Filmen und Videos aus den 7oer Jahren, dass die oft als Godards unsichtbare Phase betitelten Jahre gar nicht unsichtbar waren. Die zahlreichen positiven Besprechungen zu Numéro Deux (Jean-Luc Godard, F 1975) verdeutlichen dies.

Interessant wird es auch gerade dort, wo de Baecque sich von Godard entfernt und über andere Mitglieder schreibt, bspw. über die Mitarbeit des Lettristen Marc'O an Vent d'est (Groupe Dziga Vertov, F/I/BRD 1970) oder über Jean-Pierre Gorin, seinen engsten Mitarbeiter aus der Zeit der Groupe Dziga Vertov. ${ }^{15}$ De Baecques Biografie ist glücklichenweise keine Festschrift zum 8o. Geburtstag geworden und bemüht sich nicht, höfliche Distanz zu wahren. So hält sich der Autor beispielsweise nicht mit Kritik am gescheiterten Ausstellungsprojekt Collage(s) de France 2006 im Centre Pompidou zurück und schreibt das Scheitern mit aller Deutlichkeit Godard zu. ${ }^{16}$

\section{No portrait}

Die 20. Ausgabe der Film-Konzepte, die sich Jean-Luc Godard widmet, behandelt in einzelnen Aufsätzen verschiedene Aspekte, die sich in der Auseinandersetzung mit den Filmen Godards ergeben. Dass die Autoren damit ein «Portrait of the Artist at Eighty» (Kiefer, S. 7) entwerfen, wie in der Einleitung in Anlehnung an den Untertitel von MacCabes Biografie vorgeschlagen, ist wenig überzeugend - und auch nicht Aufgabe solch einer Auseinandersetzung. Erwarten würde man dafür aber eine Reflektion, die das bereits Geleistete auf dem Gebiet stärker in Betracht zieht und sich mehr auf die Lücken konzentriert. ${ }^{17}$ Zwar ist hier die Beschäftigung mit seinen Filmen vor und nach 1968 ausgewogener, aber die Aufsatzsammlung bietet insgesamt nur wenig Neues. ${ }^{18}$ (Der Satz «Film ist die Wahrheit, $24 \mathrm{Mal}$ in der Sekunde» wird mindestens viermal im Heft zitiert.) Dominant sind hier die Positionen, die Godard unter auteur-Gesichtspunkten diskutieren, das selbstreflexive Moment in seinen Filmen herausarbeiten oder sie innerhalb der Intermedialitätsdebatte betrachten. Weniger etablierte Impulse, wie man sie vielleicht anlässlich eines runden Geburtstags erwarten würde, sind rar. Jürg Stenzls Analyse der verwendeten Musik zu Liberté et Patrie (Jean-Luc Godard, Anne-Marie Miéville, $\mathrm{CH}$ 2002) bietet hierzu einen inspirierenden Ansatz, der auch auf seine ebenfalls 2010 erschienene Studie zur Musik in den Filmen Godards neugierig macht. ${ }^{19}$ Oder auch der Aufsatz

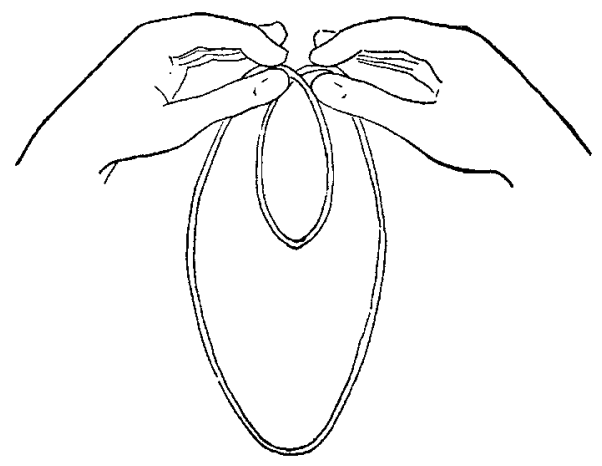

Oksana Bulgakowas zu den Cinétracts, den anonymen Kurzfilmen von 1968, ein Gegenstand, der immer noch weder einfach zu sehen noch genügend aufgearbeitet ist. ${ }^{20}$ Es sind diese weniger bekannten Perspektiven und Ansätze, die man sich bei der Auseinandersetzung mit Godard, die ja inzwischen auch ins sechste Jahrzehnt geht, deutlicher gewünscht hätte.

«ll est notre contemporain: son cinéma dure encore», schließt de Baecque seine Biografie (S.818). Und die Auseinandersetzungen mit ihm dauern auch noch an, wie die Debatten und Publikationen an und nach seinem runden Geburtstag zeigen. Die Arbeit mit seinen Filmen ist vielleicht mehr als bei anderen Filmemacherinnen und Filmemachern auf diese unterschiedlichen Bausteine ${ }^{\mathbf{2 1}}$ angewiesen. Um so enttäuschender, dass man noch immer auf eine Veröffentlichung seiner beiden Fernseharbeiten Six fois Deux - sur et sous la communication (Jean-Luc Godard, Anne-Marie Miéville, F 1976) und France/tour/détour/deux enfants (Jean-Luc Godard, Anne-Marie Miéville, F 1977) warten muss. ${ }^{22}$ So wie übrigens auch auf die der Filme von Anne-Marie Miéville, mit der er diese beiden Serien wie auch viele andere Filme realisierte.

1 Roger Ebert, Cannes\# 5 : Waiting for Godard, in: Roger Ebert's Journal, dort datiert 17.5.2010, http:|/blogs.suntimes.com/ ebert/2010/05/cannes_5_waiting_ for_godard.html, gesehen am 27.6.2011.

2 Daniel Cohn-Bendit konnte ihn nicht zum Kommen bewegen: Jean-Luc Godard à Daniel Cohn-
Bendit: «Qu'est-ce qui t'intéresse dans mon film?», in: Télérama, dort datiert 12.5.2010, http://www. telerama.fr/cinema/jean-luc-godarda-daniel-cohn-bendit-qu-est-ce-qui-tinteresse-dans-mon-film,55846.php, gesehen am 27.6.2011.

3 http://www.festival-cannes.com/ assets/Image/Direct/033108.pdf, gesehen am 27.6.2011. 
4 «He just told me, st's not the Oscars». At first he thought it was going to be part of the same ceremony, then he realised it was a separate thing in November.» Anne-Marie Miéville im Interview: Jean-Luc Godard to be an Oscar no-show, in: The Australian, dort datiert 7.9.2010, http://www. theaustralian.com.au/news/arts/jeanluc-godard-to-be-an-oscar-no-showl story-e6frg8n6-1225915014578, gesehen am 27.6.2011.

Einen amerikanischen Preis wollte er schon 1995 nicht, weil «JLG n'a pas été capable, tout au long de sa carrière de movie maker/goer, de: empêcher M. Spielberg de reconstruire Auschwitz [...]» (Antoine de Baecque, Godard, 733).

5 «lch habe den Krieg miterlebt, mir ist erst nachher aufgegangen, daß meine Eltern aus einer Familie von Kollaborateuren kamen. Mein Großvater war ein wütender - nicht etwa Antizionist, er war Antisemit, während ich Antizionist bin, er war Antisemit oder sowas. Daher kommt es, daß ich heute so viele Bücher über Hitler gelesen habe, über die Konzentrationslager, über alles das, wahrscheinlich viel mehr als irgendein Judenkind, obwohl ich persönlich keine Beziehung zu den Problemen habe». Jean-Luc Godard, Einfuihrung in eine wahre Geschichte des Kinos, Frankfurt/M. (Fischer) 1984, 56.

6 Daniel Cohn-Bendit, Mein Freund Godard, in: FAZ, 3.12.2010, http:|/www.faz.net/artikel/C $30964 \mid$ zum-achtzigsten-mein-freundgodard-30321117.html, gesehen am 30.6.2011.

7 Die Texte und Dokumente finden sich in vier Episoden auch auf der Internetseite von La Règle du Jeu: Bernard-Henri Lévy, BHL acteur de Godard? (Premier épisode), in: La Règle du Jeu, dort datiert 15.11.2010, http://laregledujeu. org/2010/11/15/3349/bhl-acteur-degodard-premier-episode|, gesehen am 28.6.2011.

8 Colin MacCabe, godard a portrait of the artist at 70 , London (Bloomsbury) 2003.

9 Die Biografie von de Baecque diskutiert - wie Richard Brody, Everything Is Cinema: The Working Life of Jean-Luc Godard, New York (Henry Holt) 2009-nur das erste Projekt, nicht aber das zu Terre Promise.
10 Auch in Lanzmanns Biografie findet sich kein Hinweis auf diese Episode (Claude Lanzmann, Der patagonische Hase: Erinnerungen, Hamburg [Rowohlt] 2010).

11 Georges Didi-Huberman hat sich mit den Positionen Lanzmanns, Godards und Wajcmans in Bilder trotz allem (München [Fink] 2007) auseinandergesetzt und Godards Position zu den Bildern der Shoah an seinen Histoire(s) du cinéma ( $\mathrm{F}$ 1998) diskutiert, wobei er es vermied, Godards dialektische Montage auf das Beispiel in Ici et Ailleurs zu reduzieren.

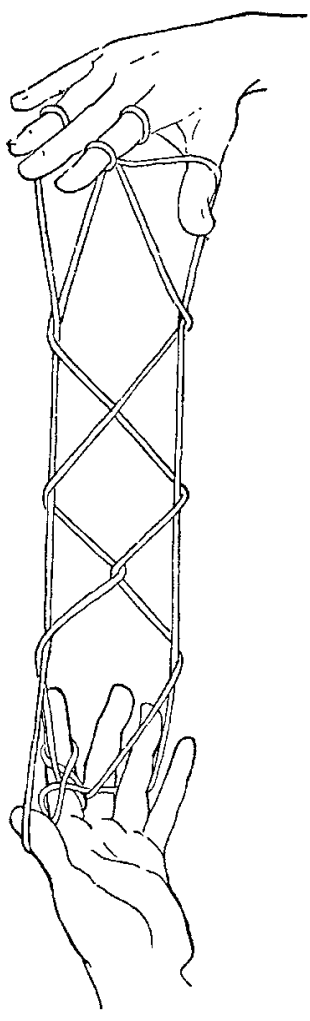

12 Alain Bergala (Hg.), Jean-Luc Godard par Jean-Luc Godard, Paris (Cahiers du cinéma) 1998.

13 «Als er [Godard] 2003 den Band Godard, a portrait of the artist at 70 von Colin MacCabe erhält und überfliegt, zerreißt er wütend die Seiten vor seinem Freund Freddy Buache. Im September 2008 schickt er Richard Brody sein Werk Everything Is Cinema. The Working
Life of Jean-Luc Godard zurück, den Einband mit schwarzem Filzstift überschrieben und dem abgewandelten und übersetzten Zitat von Victor Hugo: «As long as there will be scrawlers to scrawl, there will be murders to kill» (de Baecque S. 8f. Übers. FK).

14 De Baecque berichtet kurz von einem Interview, das das ZDF 1970 mit Godard und der Gruppe gedreht hat, im Verlauf dessen Godard eine Postkarte mit einem aus einem Davidstern geformten Hakenkreuz und der Unterschrift «Nazisrael» in die Kamera hält. Er befiehlt dem Kameramann, ihm einen Scheck auszustellen, Geld, das, da es aus Deutschland kommt, nun den Zionisten fehlen wird, wie er sagt. Anschließend befiehlt er der Gruppe, dass sich jeder kurz die Karte vor das Gesicht halten solle, was auch alle tun. Vielleicht das deutlichste Bild, nicht nur für Godards Haltung zu Israel, sondern auch dem Kollektivgedanken gegenüber. Nachzulesen bei de Baeque aufS. 531, zu sehen unter: http://www.youtube.com/ watch?v=GQsuOwq7QFU, gesehen am 29.6.2011.

15 Siehe hierzu auch das Interview mit Jean-Pierre Gorin: Twin Brother - Jean-Pierre Gorin im Gespräch, in: CARGO - Film/ Medien/Kultur, dort datiert 10.4.2011, http:||www.cargo-film.de| kino-dud/twin-brother-jean-pierregorin-im-gesprach/?highlight=gorin, gesehen am 29.6.2011.

16 Godard setzte den Leiter des Departements Dominique Païni vom Projekt ab und präsentierte 2006 eine reduzierte Version unter dem Titel Voyage(s) en Utopie (nachzulesen auch in den Cahiers du Cinéma, Heft 611 (April 2006)). Um so erstaunlicher, dass Païni dennoch eine Einführung zu dieser Ausstellung gegeben hat («ll fallait qu'on s'est sepparé, c'est une gèste de violence pour inventer.») - zu sehen auf dem Extra der DVD Morceaux de conversation avec Jean-Luc Godard.

17 Dies ist der Fall in dem Sammelband von Michael Temple, James S. Willimas (Hg.), The Cinema Alone. Essays on the Work of Jean-Luc Godard 1985-2000, Amsterdam (Amsterdam University Press) 2000. Auch hier nimmt man gleich mit dem ersten Satz Bezug auf den 70. Geburtstag Godards. Bei de Baecque findet sich der Hinweis auf den runden Geburtstag im Klappentext.

18 «Für Godard sind Frauen, das sei schon an dieser Stelle konstatiert, ein unergründliches Mysterium [...] Sie zählen [...] zu den wahren Schauwerten in den Filmen der Nouvelle vague, die ja generell präsentierten, dass Kino heißt, hübsche Frauen hübsche Dinge machen zu lassen» (Norbert Grob, Féminin-masculin. Godard und die Frauen: Miniaturen, in: Kiefer et al., 9-22, hier 1off).

19 Jürg Stenzl, Jean-Luc Godard musicien. Die Musik in den Filmen von Jean-Luc Godard, München (edition text + kritik) 2010.

20 Wie übrigens auch die Filme der Groupe Dziga Vertov, denen Volker Pantenburg im letzten Jahr erschienenen Ränder des Kinos. Godard - Wiseman - Benning - Costa (Berlin (August Verlag) 2010) ein Kapitel widmete.

21 Ein weiterer Baustein ist der Film Deux dans la vague (Regie: Emmanuel Laurent, F 2010) über das Verhältnis zwischen Godard und Truffaut. De Baecque hat dazu das Drehbuch geschrieben und der Film enthält zu diesem Thema mehr Informationen als die Biografie. Im Gegensatz zu Film socialisme kam Deux dans la vague 2011 in Deutschland in die Kinos.

22 Die Filme der Groupe Dziga Vertov sind bereits 2008 auf dem spanischen Label Intermedio auf DVD mit spanischen Untertiteln erschienen. 